\title{
Economic Optimization Operation Analysis of Microgrid Based on Improved Particle Swarm Optimization
}

\author{
Jialiang Ling ${ }^{1,2}$, Jiannan $\mathrm{Li}^{1}$, Di Feng ${ }^{3}$ and Youwen Tian ${ }^{1, *}$ \\ ${ }^{1}$ College of information engineering, shenyang agricultural university, shenyang 110866, China \\ 2State Grid Anshan Power Supply Company, Anshan 114001, China \\ ${ }^{3}$ Liaoning radio and television group, shenyang 110004 , China \\ *Corresponding author
}

Keywords: Micro grid, Economic optimization, Improved particle swarm optimization.

\begin{abstract}
In view of the problem of optimal operation of microgrid system, a mathematical model of economic optimal dispatch of microgrid was established in this paper, aiming at the lowest cost of generating electricity of distributed generation, operation and maintenance of corresponding equipment, and the lowest price of purchasing and selling electricity from large power grid. And the three kinds of operation scheduling under different weather conditions (normal, cloudy, sunny) were analyzed respectively. Finally, improved particle swarm optimization (iPSO) algorithm was improved to optimize the scheduling under the constraints of not only load requirements, but also generation and output constraints, energy storage unit operation constraints and interactive energy with large power grids. The results show that the proposed control strategy can effectively achieve the optimal power distribution of microgrid and minimize the operation cost.
\end{abstract}

\section{Introduction}

New energy technologies have achieved adequate development and improvement under the policy support in recent years, and achieved fruitful results in solving the problems of national economy, energy shortage and environmental pollution. Nowadays, as the main carrier of wind power generation, photovoltaic power generation, micro-gas turbine and energy storage battery, microgrid has been widely developed and applied[1]. How to properly deal with the economic operation of the combination of different new energy equipment to achieve economic optimization has become a new research topic. In this paper, improved particle swarm optimization (pso) algorithm is proposed to study the optimal operation of micro grid economy. At the end of this paper, three typical daily weather conditions were listed, and three different optimization results were obtained by improving particle swarm optimization algorithm to provide scientific and reliable support for relevant departments to carry out power grid dispatching and formulate operation plans.

\section{Economic Optimization of Microgrid}

\section{Objective Function}

Microgrid contains a lot of distributed power supply, which includes the wind turbine, photovoltaic power genera- tion unit, the micro gas turbine unit, storage device. In order to make the economy better, the distributed power generation costs, the battery loss of the work efficiency due to often the depth using, the corresponding equipment operation mainten -ance cost and purchase and sale electricity price were consi- dered from the large power grid in this study [2].The objective function optimization of economic operation of microgrid is as follows:

$$
\operatorname{minC}(\mathrm{P})=\lambda_{\mathrm{i}} F_{\alpha i}+\lambda_{\mathrm{i}} F_{\beta i}+\lambda_{\mathrm{i}} F_{\gamma i}+C_{b}(t) \times P_{b u y}(t)-C_{s}(t) \times P_{\text {sell }}(t)
$$

Where, $C_{b}(\mathrm{t})$ and $C_{s}(\mathrm{t})$ are the purchase and sale prices of the power grid at the $t$ moment respectively; $P_{b u y}(\mathrm{t})$ and $P_{\text {sell }}(\mathrm{t})$ are the purchasing and selling power of power grid at any time respectively; $\lambda_{1}, \lambda_{2}, \lambda_{3}$ are the weight ratios of cost, pollution gas penalty cost and operation and 
maintenance cost respectively; $F_{\alpha i}\left(\mathrm{P}_{D G i}\right)$ is the cost of the distributed power generation No.i; $F_{\beta i}\left(\mathrm{P}_{D G i}\right)$ is the penalty cost of the distributed power supply pollution gas emission No.i; $F_{r i}\left(\mathrm{P}_{D G i}\right)$ is cost for the first distributed power generation equipment operation and maintenance; $\mathrm{C}(\mathrm{P})$ is the total power generation cost of the system.

\section{Constraints}

The grid-connected operation of microgrid must meet certain constraint conditions, including node power flow constraint, power balance constraint, distributed unit power generation output constraint, node voltage constraint, energy storage unit operating state constraint, and interaction capacity constraint with large grid.

1) Power balance constraint

In any dispatching period, the electric power balance constraint must be satisfied. Electrical power shall meet the following requirements:

$$
\sum_{i=1}^{N} P_{\text {gen }, i}+P_{\text {buy }}(\mathrm{t})-\mathrm{P}_{\text {sell }}(t)=P_{\text {Load }}
$$

Where, $P_{g e n, i}$ is the power generation output of each distributed generation unit within any period of time, which may be one or several of wind power generation output, photovoltaic power generation output and energy storage battery power output; $P_{\text {Load }}$ is all power loads in the micro grid; $P_{b u y}$ is the power purchased from the large grid; $P_{\text {sell }}$ is the power sold to the large grid.

2) Power Generation Output Constraint

The inequality constraint of the power generation output constraint is:

$$
P_{D G i}^{\min } \leq P_{D G i} \leq P_{D G i}^{\max } \quad(\mathrm{i}=1,2, \cdots, \mathrm{N})
$$

Where, $P_{D G i}^{\min }$ and $P_{D \text { ma }}^{\max }$ are respectively the minimum and maxi- mum safety outputs of the first distributed generation unit.

3) Operating Constraints of Energy Storage Units

For the battery, it must meet its load state constraint in any operating period, and this paper assumes that the battery reaches the balance of charge and discharge in one day, so as to ensure a large scheduling space in the next scheduling cycle. Operating constraints of energy storage units are expressed as follows:

$$
\begin{aligned}
& P_{S}^{\min } \leq P_{S} \leq P_{S}^{\max } \\
& E_{S}^{\min }<E_{S}(\mathrm{t})<E_{S}^{\max } \\
& \frac{1}{\eta_{D}} \sum_{P(\mathrm{k})>0, k=1}^{T} P_{S}(\mathrm{k})+\eta_{\mathrm{C}} \sum_{P(k)<0, k=1}^{T} P_{S}(\mathrm{k})=0
\end{aligned}
$$

Where, $P_{\mathrm{S}}^{\max }$ and $P_{\mathrm{S}}^{\min }$ are respectively the maximum and mini- mum charge-discharge powers of the battery; $E_{s}{ }^{\min }$ and $E_{s}{ }^{\max }$ are respectively the maximum and minimum residual capacity of the battery. $\eta_{D}$ is charging efficiency; $\eta_{C}$ is discharge effic- iency;Equation (6) indicates that the charging and discharging energy of the battery can reach equilibrium in a dispatching cycle.

For thermal energy storage device, its storage energy and charging and discharging rate must meet certain limits in any operating period [3], as shown in the following equation:

$$
\begin{aligned}
& Q_{S_{t h}}^{\min } \leq Q_{S_{t h}}(\mathrm{t}) \leq \mathrm{Q}_{S_{s_{h}}^{\max }} \\
& P_{S_{t h}}(\mathrm{t}) \leq P_{\text {th_dech }}^{\max } \quad\left(P_{S_{s_{h}}}(\mathrm{t})>0\right) \\
& -P_{S_{t h}}(\mathrm{t}) \leq P_{\text {th_dech }}^{\max } \quad\left(P_{S_{t h}}(\mathrm{t})<0\right)
\end{aligned}
$$


In the above equation, $Q_{S_{t}}$ is the energy storage state of thermal energy storage device in time period t, $P_{S_{\mu}}(\mathrm{t})$ is the charge and discharge power of thermal energy storage device in time period $\mathrm{t}$, $Q_{s_{n}}^{\max }\left(Q_{s_{n}}^{\min }\right)$ is the maximum and minimum energy storage capacity of thermal energy storage device, and $P_{t_{\text {thch }} \max }^{\left(P_{\text {th_dech }}^{\max }\right)}$ is the maximum charge and discharge power of thermal energy storage device.

4) Interaction capacity constraint with large power grid

The maximum capacity of energy interaction between micro grid and large grid shall not exceed the limit of physical transmission capacity of tie lines or the supply and demand agreement reached by them:

$P_{\text {Line }}^{\min } \leq P_{\text {Line }} \leq P_{\text {Line }}^{\max }$

Where, $P_{\text {Line }}^{\max }$ and $P_{\text {Line }}^{\min }$ are the upper and lower limits of energy interaction between microgrid and large grid respectively.

\section{Scheduling Strategy}

All the generating units in the microgrid and the large grid participate in the optimal dispatching together. When the power required by the microgrid is insufficient, the microgrid can obtain power from the large grid and output power to the large grid when the power generated by the microrid is surplus [4].

\section{Improved Particle Swarm Optimization Algorithm}

\section{Basic Principles of Particle Swarm Optimization}

Like other evolutionary algorithms, particle swarm optimization (PSO) searches for the optimal solution through continuous iteration in a group of initial solutions generated by machines [5, 6].

Assuming that the number of particles in the particle swarm is $\mathrm{N}$, the D-dimensional space coordinate and speed about each particle at time t.

$$
\begin{aligned}
& \overline{x_{i}}(\mathrm{t})=\left(\mathrm{x}_{i}^{1}, \mathrm{x}_{i}^{2}, \cdots, \mathrm{x}_{i}^{d}, \cdots, \mathrm{x}_{i}^{D}\right) \\
& \overline{v_{i}}(\mathrm{t})=\left(\mathrm{v}_{i}^{1}, \mathrm{v}_{i}^{2}, \cdots, \mathrm{v}_{i}^{d}, \cdots, \mathrm{x}_{i}^{D}\right)
\end{aligned}
$$

At $\mathrm{t}+1$, the coordinate position ${\overline{x_{i}}}_{i}(t)$ and velocity $\bar{v}_{i}(t)$ are adjusted in the manner described in formula (13) and formula (14).

$$
\begin{aligned}
& \bar{v}_{i}(\mathrm{t}+1)=\bar{v}_{i}(\mathrm{t})+c_{1} \cdot r_{1}\left(\bar{p}_{i}(\mathrm{t})-\overline{x_{i}}(\mathrm{t})\right)+c_{2} \cdot r_{2}\left(\overline{p_{g}}(\mathrm{t})-\bar{x}_{i}(\mathrm{t})\right) \\
& \bar{x}_{i}(\mathrm{t}+1)=\bar{x}_{i}(\mathrm{t})+\bar{v}_{i}(\mathrm{t}+1) \\
& \begin{cases}v_{i}^{d}=v_{\max }, \quad \text { if } & v_{i}^{d}>v_{\max } \\
v_{i}^{d}=-v_{\max }, \quad \text { if } & v_{i}^{d}<-v_{\max }\end{cases}
\end{aligned}
$$

Where, $c_{1}$ and $c_{2}$ represent the learning constant of particle, respectively. While ${ }^{r_{1}}$ and ${ }^{r_{2}}$ get values uniformly between 0 and $1 ; \mathrm{P}_{\mathrm{i}}$ is the best position of particles individual experienced, while $P_{g i}$ is population has experienced the best position. When particles in iterative process, particle velocity may exceed a maximum set, formula (15) is used to ensure particle speed within a certain range.

\section{Improved Particle Swarm Optimization}

Particle swarm optimization (pso) has a fast convergence speed in the early optimization process, but it has some disadvantages, such as low accuracy, easy dispersion. In addition, the convergence speed of the algorithm reduce in the later stage, and also affects the optimization ccuracy. Therefore, 
inertia weight $\omega$ is introduced to improve the convergence speed and accuracy in this paper [7].The update formula of velocity after introducing the inertia weight is:

$$
\bar{v}_{\mathrm{i}}(\mathrm{t}+1)=\omega \bar{v}_{\mathrm{i}}(\mathrm{t})+\mathrm{c}_{1} \cdot \mathrm{r}_{1}\left(\bar{p}_{\mathrm{i}}(\mathrm{t})-\bar{x}_{\mathrm{i}}(\mathrm{t})\right)+\mathrm{c}_{2} \cdot \mathrm{r}_{2}\left(\bar{p}_{\mathrm{g}}(\mathrm{t})-\bar{x}_{\mathrm{i}}(\mathrm{t})\right)
$$

The inertia weight $\omega$ itself is a linear change parameter related to the number of iterations (value range $0 \sim 1$ ). Its role is that $\omega$ value is relatively large when early convergence speed is fast so that the particle search in a large range of range area, which achieve the approximate optimal value position can be positioned quickly. As the number of iterations increases, the convergence rate gradually slows down, and $\omega$ value decreases linearly, which increase the local search accuracy. Linear change formula of inertia weight is:

$$
\omega=\omega_{\max }-\frac{\omega_{\max }-\omega_{\min }}{T_{\max }} \times T_{n}
$$

Where $T_{n}$ is the current number of iterations, $\omega_{\max }$ and $\omega_{\min }$ are the maximum and minimum values of weights, and $T_{\max }$ is the maximum number of iterations.

\section{Solution Method of Microgrid Optimization}

\section{Optimization Methods and Analysis}

In this study, the dispatching strategy of grid-connected microgrid is adopted, that is, the interconnection of microgrid and external large-scale grid can realize the two-way flow of energy [8]. When the microgrid generates too much electricity, it can sold to the external grid to get profit.When the energy generated by the microgrid is too low, the electric energy must be purchased from the external grid to supplement the insufficient power supply. When the microgrid allows two-way electric energy interaction with the main network and adds peaking and valley filling measures, the operation economy of the microgrid system will be significantly improved $[9,10]$.

The improved particle swarm optimization algorithm was adopted to microgrid economic operation optimization scheduling problems analysis calculation. That is the problem of solution minimum value applying improved particle swarm optimization algorithm under the relevant equality constraints and inequality constraints. The penalty coefficients were added to the objective function in this paper when dealing with constraints.

\section{Steps of Optimization Algorithm}

a) Initialize the system and set constraints of optimization variables.

b) Given the algorithm parameters, $\mathrm{Max}^{\mathrm{dt}}=500$ (the maxi- mum number of iterations is 500 ); $\mathrm{D}=72$ (number of spatial dimensions); $\mathrm{N}=600$ (the number of particles is 600 ); $\omega_{\max }=0.9$ (maximum inertia weight); $\omega_{\min }=0.4$ (minimum inertia weight).

c) Calculate the generation and consumption of electricity in each period of the research cycle.

d) Calculate the objective function and adaptive value of each particle. Then compare its adaptive value with the historical optimal value, and select the optimal value as the solution of this particle. The global optimal solution can be obtained.

e) Update the inertia weight, position and velocity of particles to form a new generation of particles.

f) When the maximum number of iterations is reached, the algorithm can be quit, and the output result is the operation plan in the most economical situation.

\section{Simulation Results and Case Analysis}

According to the previous proposed comprehensive cost objective function and scheduling strategy, combining constraints. The improved optimization objective function is:

$$
\begin{aligned}
& \operatorname{minC}(\mathrm{P})=\lambda_{\mathrm{i}} F_{\alpha i}+\lambda_{\mathrm{i}} F_{\beta i}+\lambda_{\mathrm{i}} F_{\gamma i}+C_{b}(t) \times P_{b u y}(t)-C_{s}(t) \times P_{\text {sell }}(t)+d \times \Delta P_{B A}+r \times \Delta P_{\text {sum }}+ \\
& e \times \Delta P_{D G}+h \times \Delta P_{\text {Line }}
\end{aligned}
$$


Where the $\Delta P_{B A}$ is the value of subtraction between cumulative charging capacity and rated capacity of energy storage unit; $\Delta P_{\text {sum }}$ is the value of the difference between power generation and power consumption load; $\Delta P_{D G}$ is the difference between the minimum and maximum safety output of distributed generation; $\Delta P_{\text {Line }}$ is the difference between the transmission capacity of micro-grid and large-grid; $d, r, e$ and $h$ are all penalty coefficients[11].

In this paper, three typical daily weather conditions were selected in certain winter, the generation cost of microgrid was research object to optimize the control of in the next 24 hours, that is, the simulation period was 24 hours.

a) Typical Days of Normal Weather: power curve of new energy equipment of microgrid within 24 hours of the day is shown in Fig.1. In Fig.1, the MT curve is the output of micro-gas turbine. The GRID curve is the electric quantity exchanged between large GRID and micro GRID. The BA curve is the charge and discharge of the battery (charge is negative, discharge is positive); The MT curve is the power generation of micro gas turbine. The ODD curve is optimal power after the micro-gas turbine machine meet the heat load. It is stipulated that the purchase of electricity from the large power grid is represented by a positive number, and the sale of electricity to the large power grid is represented by a negative number. It is known from Figure.1:

In the first period from 0:00 to 7:00: electricity load was small, and the light intensity in this period was 0 , so the output power of photovoltaic power generation was 0 , and local wind turbines can be used to generate electricity.

The the second time period was from 7:00 to 10:00: during this period, the power load gradually increases and the light intensity gradually increases from 0 , but the power generation is less and needs additional supplement.

The the third time period was from 10:00 to 19:00: the power load reached its peak, and the output of photovoltaic units and wind turbines almost reached the maximum.

The fourth time period between 21:00 and 24:00: electricity load was reduced, and the light intensity was 0 during this period, so the wind turbine can be used to generate electricity in priority. When the electricity generated by the wind turbine was insufficient, it can be supplied through the external large power grid.

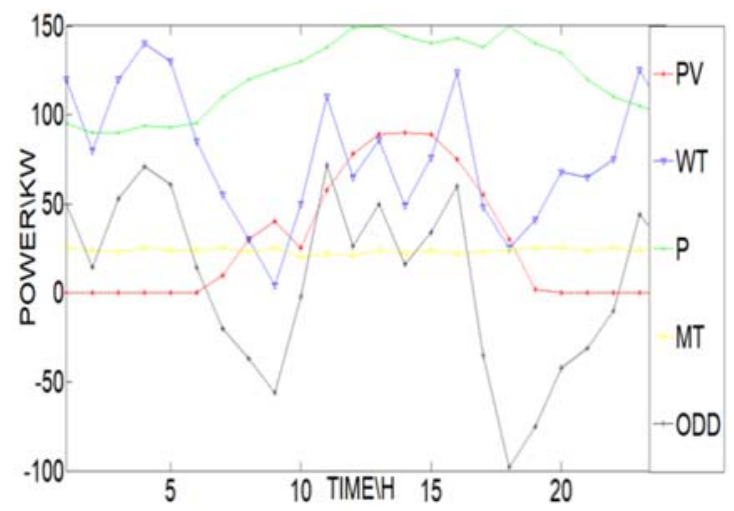

Figure 1. Power diagram of new energy in normal weather

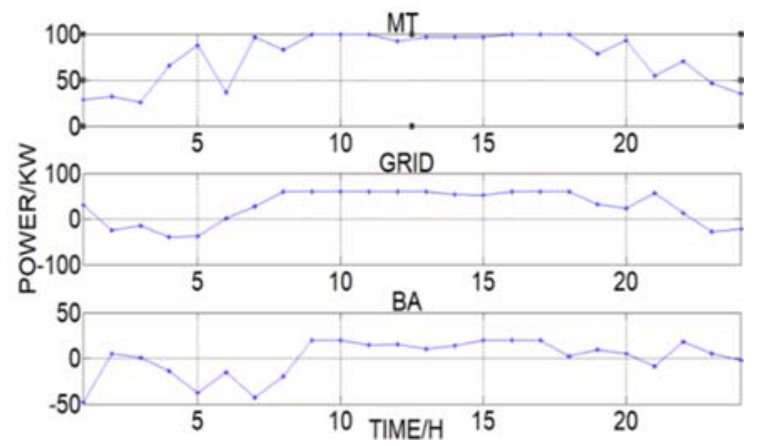

Figure 2. Optimal dispatching diagram of microgrid equipment in normal weather

In this paper, improved particle swarm optimization algorithm was adopted to optimize the 
scheduling of economic operation of microgrid, and the results were shown in Figure.2. It can be seen from the Figure.2 that during 0:00 to 8:00, the user needed less electricity, and the energy storage device was mainly charged. After 8:00, the user load increased, and the energy storage equipment was mainly discharging. In the period 0:00-6:00 and 23:00-24:00, the microgrid system generated more electricity than the user, then sell surplus electricity to power grid to obtain better economic benefits. Between 6:00 and 22:00, when the battery and other generating units working together also couldn't satisfy the user's electricity, purchasing power was needed from the power grid to make up for the lack of electricity. According to this optimization strategy, the microgrid can achieve the most economical.

b) Typical conditions on cloudy days: on cloudy days, photovoltaic power generation was reatively small, and the corresponding dispatching of power generation of micro-gas turbine, electrical energy exchanged with large power grid, and dispatching of storage battery were needed to make up for the shortage of photovoltaic power generation. The power curve of new energy equipment of microgrid within 24 hours on a day was shown in Figure. 3. It could be seen from the power comparison between Figure. 3 and Figure.1 that when PV generates less power due to weather, the curve of residual power changes greatly. In order to make the microgrid meet the best economic requirements, MT operation plan, GRID operation plan and BA operation plan should be changed accordingly.

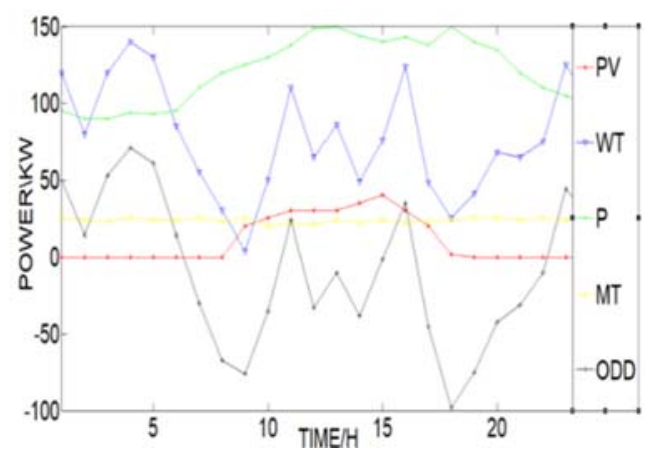

Figure 3. Power diagram of new energy equipment on cloudy days

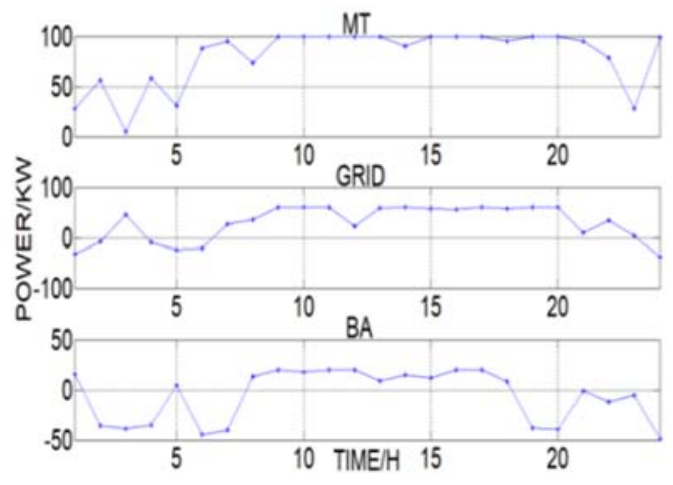

Figure 4. Optimal dispatching diagram of microgrid on cloudy days

The improved particle swarm optimization algorithm was adopted to optimize the scheduling of economic operation of microgrid with cloudy days, and the results were shown in Figure.4. By comparing Figure.4 with Figure.2, we can see that the electricity of microgrid selling to the large power grid should be reduced correspondingly during the period from 0:00 to 8:00, and the charging of energy storage equipment should be increased at the same time. During the period from 10:00 to 18:00, the battery should discharge more to meet the normal load. In the case of less photovoltaic power generation in cloudy days, MT operation plan, GRID operation plan and BA operation plan could make the microgrid the most economical under the operation strategy of Figure.4.

c) Typical sunny days: when the weather was sunny, the photovoltaic power generation was high, and the corr- esponding dispatching micro-gas turbine power generation, electrical energy exchanged with the large power grid, and dispatching battery energy storage condition meet the 
operation optimization of the microgrid [12-13]. The power curve of microgrid new energy equipment within 24 hours of a day when the weather was good was shown in Figure. 5.

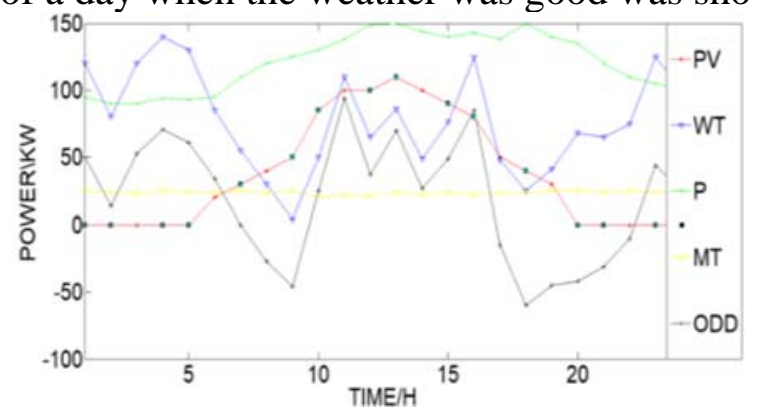

Figure 5. Power diagram of sunny new energy equipment

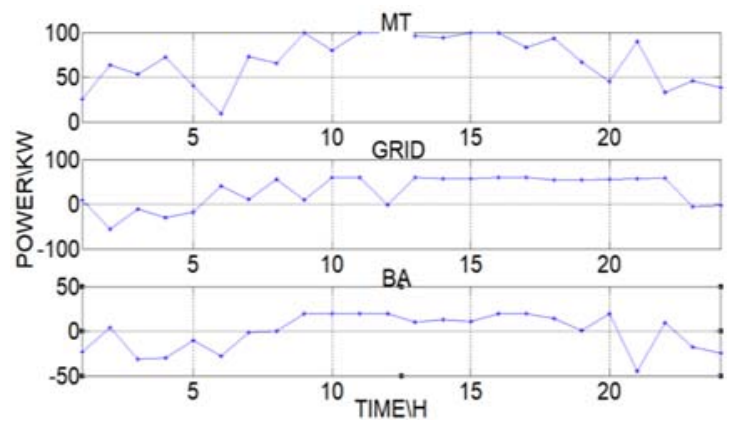

Figure 6. Optimal dispatching diagram of sunny microgrid

An improved particle swarm optimization (PSO) algorithm was used to optimize the economic operation of microgrid with more photovoltaic power generation in sunny weather. The results are shown in Figure.6. By comparing Figure.6 with Figure.2 and Figure.4, it could be seen that the MT generation plan, GRID generation plan and BA operation plan had changed with the change of photovoltaic power generation. In order to optimize the economy of the microgrid when the generation and load of the microgrid are changing, a reasonable operation plan can be arranged by improving the particle swarm optimization algorithm, so as to optimize the economy of the microgrid.

\section{Conclusion}

The research of microgrid in China is still in its infancy. The research of microgrid economy is not deep enough. There are still many basic works to be completed. A mathematical model for optimizing the operation of microgrid system through economy was established in this paper, including wind power generation, photovoltaic power generation, micro-turbine power generation, and power exchange with large grid. The penalty factor was introduced into the objective function to make it an optimization problem of single objective. The complexity of the algorithm is effectively reduced when the improved particle swarm optimization algorithm is used to optimize the economic operation of the microgrid system. Finally, combined with the optimal dispatching comparison under three different weather conditions, it could be found that when the output of distributed generation changes, the economy of microgrid could be optimized through different operation plans.

\section{References}

[1] Wu Ding, Gao Cong, Ji Zhicheng. "Economic optimization operation of the microgrid using the hybrid particle swarm optimization algorithm,”. Control Theory and Application, vol. 35,pp. 457-467,2018.

[2] Shujian Zhao, Chenglian Ma, Li Sun, Yuanzhu Meng, Wei Wu, Luheng Huang. "Genetic Algorithm Based Economic Optimization of Microgrid Including Multi-Energy Supply System,” 
Journal of Physics: Conference Series, vol. 1072, pp.1-6,2018.

[3] Jin Shaozhen, Mao Zhizhong and Li Hongru. "Economic operation optimization of smart microgrid considering the uncertainty”. Control theory and Application, vol. 35,pp.1357-1370,2018.

[4] Cheng Qiming, Cheng Yinman, Huangshan, Zhang Qiang. “Summary on Economic Optimal Operation of Micro-grid”. Guangdong Electric Power, vol. 31,pp.1-9,2018.

[5] Huang Chen, Zhang Bianshe. "Micro Grid Scheduling Optimization Based on Quantum Particle Swarm Optimization(PSO)Algorithm ” Power Grid and Clean Energy,vol.32 ,pp.72-76,2016.

[6] Cheng Yuxu. " Research and Implementation of Microgrid Energy Optimal Dispatching Based on Improved Particle Swarm Optimization,” Central South University, 2013.

[7] Gao Cong, Wu Ding, Pan Tinglong, Ji Zhicheng. "Microgrid economic operation optimization based on immune particle swarm optimization," Journal of System Simulation,vol.30,pp. 636-646,2018.

[8] Sachidananda Sen, Vishal Kumar. “Microgrid modelling: A comprehensive survey,”. Annual Reviews in Control, vol.46, pp. 216-250,2018.

[9] Yang Xinfa, Su Jian, Lu Zhipeng, Liu Haitao, Li Rui. “Overview on Micro-grid Technology,” Journal of Electrical Engineering of China, vol. 34,pp. 57-70,2014.

[10] Chen Hong, Lenghua, Tang Haiguo, Zhu Jiran, Kang Taifeng and Zhong Huabing. "Two-stageoptimization method on micro-grid economic operation with renewable energy resources,” Journal of Electric Power Science and Technology, vol. 33, pp.93-98,2018.

[11] Xiaoqian Zhou, Qian Ai,“A distributed economic control and transition between economic and non-economic operation in islanded microgrids,” Electric Power Systems Research, vol.158,pp.70-81,2018.

[12] LI Lingyi. "Research on the control method under the mode of grid-connected operation of microgrid,”. Innovation and application of science and technology, vol.14,pp.132-133,2019.

[13] Faisal R. Badal, Purnima Das, Subrata K. Sarker, Sajal K. Das. “A survey on control issues in renewable energy integration and microgrid,” Protection and Control of Modern Power Systems, vol.4, pp.1-24,2019. 